\title{
The Effects of Palatable Sweet Ingesta on Human Responsivity to Heat Pain
}

\author{
Michele E. Mercer ${ }^{1 *}$, Mark D. Holder'2, Russell J. Adams ${ }^{1,3}$ \\ ${ }^{1}$ Department of Psychology, Memorial University of Newfoundland, St. John's, Canada \\ ${ }^{2}$ Department of Psychology, University of British Columbia, Kelowna, Canada \\ ${ }^{3}$ Discipline of Pediatrics, Faculty of Medicine, Memorial University of Newfoundland, St. John's, Canada \\ Email: "michelem@mun.ca, mark.holder@ubc.ca
}

Received 14 July 2015; accepted 6 September 2015; published 10 September 2015

Copyright (C) 2015 by authors and Scientific Research Publishing Inc.

This work is licensed under the Creative Commons Attribution International License (CC BY).

http://creativecommons.org/licenses/by/4.0/

(c) (i) Open Access

\section{Abstract}

It is well known that the taste of sweet solutions produces a morphine-like analgesia in both rats and human infants, and under certain conditions, possibly in human adults. To further explore whether ingestion analgesia persists into human adulthood, the present study was the first to utilize contact heat, a method of pain induction used commonly in both behavioural and pharmacological studies with laboratory animals. Left arms of 120 university undergraduates were exposed to a hot-plate, with pain responsivity assessed both before and after consuming either nothing (control group), or foods that they rated previously as unpalatable (e.g., black olives), neutral (e.g., rice cakes), or palatable (e.g., chocolate-chip cookies). Pain responsivity was assessed with four pain measures: pain threshold, pain tolerance, and visual analogue scale (VAS) ratings of pain intensity and unpleasantness. Between-groups comparisons in 2 separate experiments revealed that women (but not men) who consumed a palatable food showed increased pain tolerance, relative to the nothing, unpalatable, or neutral groups. Collectively, these data support our previous findings that "palatability-induced analgesia" exists in human adults, at least in females. Moreover, the findings support contact heat as a suitable method for assessing ingestion analgesia to experimental pain with human adults.

\section{Keywords}

Heat Pain, Analgesia, Palatability, Sweet Ingestion, Humans, Opioids

\section{Introduction}

It is now well established that the consumption of sweet ingesta increases endogenous opioid peptide (EOP) ac-

\footnotetext{
${ }^{*}$ Corresponding author.
}

How to cite this paper: Mercer, M.E., Holder, M.D. and Adams, R.J. (2015) The Effects of Palatable Sweet Ingesta on Human Responsivity to Heat Pain. Journal of Behavioral and Brain Science, 5, 405-419. 
tivity in rat brain, plasma and cerebral spinal fluid, as well as in human plasma [1]-[4]. Given that EOPs are known to decrease pain, researchers have been very interested in establishing whether sweet ingestion can play an important role in influencing pain responsivity [5]-[7]. Experimental studies show that intraoral sweet solutions (either dextrose/saccharin, or sucrose) increase rats' paw-lift latencies from a hot-plate [8]-[10], an analgesic effect resembling that produced by morphine. Moreover, this "sweet-induced" analgesia (SIA) is reversed by minimal doses of an opioid antagonist (e.g., naltrexone, naloxone), thus suggesting that EOPs are primarily responsible for mediating the analgesic effect [8] [9] [11].

Sweet intake appears to produce analgesia in human infants as well [12]-[15]. For example, as little as $2 \mathrm{ml}$ of a $12 \%$ sucrose solution markedly reduces crying in newborn infants during both circumcision and heel lance procedures [13]. The rapid onset of the analgesic effect suggests that it is produced by the sweets' pleasant taste rather than by its chemical composition or by some post-ingestive factors [4] [12]-[16]. Furthermore, a variety of preferred sweet solutions other than sucrose (e.g., aspartame, fructose, glucose) have also proven to be effective in producing analgesia in human infants [12] [17] [18]. Similarly, rat pups who consumed one of a variety of other preferred foods (e.g., milk, corn oil, polycose, or chocolate) also showed reduced pain responsivity [19]-[22]. Collectively, these findings support the hypothesis that it is the pleasant or desirable taste of the ingesta that produces the analgesic effect, as opposed to its specific sugar or caloric content per se.

At present, several researchers are examining whether this relationship between sweets and analgesia also exists in human adults. Of the few studies which have investigated SIA in adulthood, most have used the cold pressor test (CPT) [5] [7] [23] [24]. However, the results have been equivocal. For instance, Pepino and Mennella [24] found that sucrose increased the pain threshold and tolerance of 5 - $10 \mathrm{yr}$-old children, but it did not influence their mothers' pain sensitivity. A recent study by Kakeda and Ishikawa [23] reported that after ingestion of a $24 \%$ sweet solution, CPT pain thresholds increased in adult males, but not in females. Interestingly, however, they found that there was no effect on pain tolerance, and concluded that this might have been due to a ceiling effect, as subjects were limited to only 3 minutes of exposure with more than one third of the sample enduring the full duration. In a more recent study with male university students, Mercer and Holder [7] attempted to reduce the likelihood of ceiling effects by using the CPT and a longer exposure time (5 minutes), yet were unable to find evidence of sweet-induced analgesia. Thus, the CPT may not be the most suitable method for assessing analgesia, as the unique properties of cold pressor pain (e.g., its cyclical nature, numbing effects) can lead to both inflated pain measures and ceiling effects.

Collectively, these findings from CPT studies suggest that sweet-induced analgesia may occur in human adults, but it may be limited by a number of experimental parameters, namely subject gender, method of pain induction, and specific pain measures. There are few experiments that have used methods other than the CPT to explore SIA in human. One exception is a study [6] which used pressure algometry, a pain induction method used previously in SIA experiments with animals. The study also adopted the unique approach of examining the effect of palatability by using more real world ingesta (e.g., chocolate chip cookies) compared to those used in traditional SIA experiments (simple sucrose solutions). The results showed that ingesting a highly palatable sweet food produced increased pain tolerance, compared to ingesting either nothing, or foods rated either as unpalatable (black olives) or as neutral (rice cakes). Moreover, food palatability ratings were correlated with preto post-treatment changes in pain tolerance, thus indicating the importance of palatability in producing human SIA. This suggests that the effect may perhaps be more accurately described as a taste- or palatability-induced analgesia. Note, however, that this effect was demonstrated in females only. Again, as with the CPT, the lack of SIA in males may be due to limitations within the experimental design as $33.3 \%$ of men (vs. only $6.7 \%$ of women) withstood the maximum amount of pressure. As with the CPT paradigm, this apparent ceiling effect may have precluded any analgesic effects, at least for men.

The present study is designed for two reasons, namely to use an alternative method to determine whether SIA occurs in human adults, and to examine more specifically, the effects that palatability has on the relationship between ingestion and analgesia. Interestingly, researchers examining human pain responsivity have yet to utilize the method first used to demonstrate SIA in rats, namely contact heat [9] [10]. Therefore, in an attempt to better bridge current human work with the seminal work conducted with animals, the present study employs contact heat (i.e., a laboratory hot-plate) as the method of pain induction. We also use the novel strategy of employing a number of different experimental groups, each differing in the palatability of the treatment food ingested. The expectation of this approach is that it will provide us with a better understanding of the environmental conditions which regulate pain and of our internal pain-modulatory systems. Such information is also vi- 
tal to the current clinical goal of providing new and more effective approaches to the therapeutic treatment of human pain.

\section{EXPERIMENT 1}

In previous work with both cold and pressure modes of pain [6] [7], we were able to demonstrate SIA in women, but not in men. Therefore, the strategy in this first experiment is to maximize the possibility of observing SIA by limiting participants to the female gender only. If we do find that the method of contact heat yields feasible results with women, we will then conduct a more extensive study with both males and females.

\section{Material and Methods}

\subsection{Subjects}

The subjects were 40 Caucasian, right-handed, non-smoking, pain-free female university students ( $\mathrm{M}$ age = 20.7; range $=18$ - 38). An additional 2 subjects were tested but not included in the final sample; 1 because she did not follow proper procedural instructions, and 1 because she kept her forearm on the hot-plate at the maximum temperature of $48.0^{\circ} \mathrm{C}$ during the pre-treatment trial (i.e., she showed the maximum tolerance). Subjects were recruited by signs placed around campus and then contacted by phone and briefed about the experimental procedure. Prospective subjects were asked to abstain from alcohol and analgesics during the test day, and from eating or drinking anything for at least 2 hours prior to the experimental session. This duration for food deprivation was used so that subjects did not consume palatable foods prior to baseline testing. Longer deprivation periods were not used in an attempt to avoid deprivation-induced analgesia [25]. Only non-smoking, right-handed subjects were chosen because smoking reduced pain sensitivity [26], and because left limbs showed greater pain sensitivity than right limbs regardless of hand preference [27]. The experimental protocol was approved by the university's Committee for Ethics in Human Research.

\subsection{Apparatus}

A laboratory hot-plate (Socrel corp. model DS37) was used to apply contact heat to the subjects' left forearm. The hot-plate consists of a $20 \times 20 \mathrm{~cm}$ metal plate connected to a variable DC power supply. The apparatus displays the surface temperature of the metal plate in digital $0.1^{\circ} \mathrm{C}$ increments. Once the hot-plate is turned on, the temperature of the metal plate increases at a consistent rate of $1.0^{\circ} \mathrm{C}$ per $15 \mathrm{~s}$ until it reaches $48.0^{\circ} \mathrm{C}$. To evaluate whether ingestion modulates the pain system exclusively, and/or has more general effects on related sensory systems, tactile thresholds were measured with a 20-monofilament Von Frey kit (Stoelting, Co., Wood Dale, IL). A curtain was used to shield the subjects from both the hot-plate and the monofilaments.

\subsection{Procedure}

Prior to the laboratory session, subjects were contacted by phone and asked to rate the palatability of 10 foods on a 10-point scale with 1 indicating "Strongly Dislike" and 10 indicating "Strongly Like". It is interesting to note that the ratings for many of foods, including all of those targeted for the study, were highly consistent among subjects. Because of this commonality of hedonic response, subjects were assigned in pseudorandom fashion to one of four treatment groups: "Palatable" (composed of 10 Ss who all rated chocolate chip cookies between 8 and 10), "Neutral" (composed of 10 Ss who all rated rice cakes between 4 and 6), "Unpalatable" (composed of 10 Ss who all gave black olives a rating of 1 or 2 ), or "Nothing" ( $n=10$; composed of every $4^{\text {th }}$ subject selected, irrespective of her food ratings. Both pilot work and our previous studies [6] [7] revealed that these sample sizes ( $\mathrm{n}=10$ per group) provide sufficient statistical power to detect an experimental effect.

For each group, a within-subjects design was used in which both pain and touch sensitivity of each subject were measured three times (i.e., during familiarization, pre-treatment, and post-treatment). However, the familiarization trial was excluded from the data analyses as this trial served to warm up each subject's arm to a similar temperature prior to collecting data, as well as to give the subjects practice with the procedure and to ensure that they understood all of the instructions.

Subjects were tested individually in the laboratory. The experimenter, who was blind to the hypothesis of the experiment, first described the pain intensity and unpleasantness visual analogue scales (VASs) using the instructions and auditory analogy described originally by Price, McGrath, Rafii, and Buckingham [28]. Each scale 
consisted of a $10 \mathrm{~cm}$ vertical line subdivided into 20 units ranging from either "no sensation" to "most intense that one can imagine" (the pain intensity VAS) or from "not bad at all" to "most unpleasant that one can imagine" (the pain unpleasantness VAS). To familiarize the subjects with the VASs and to ensure that they were not experiencing any discomfort prior to the experiment, subjects were asked to rate their current level of discomfort using the VASs.

Testing proceeded in 4 phases: Familiarization, Pre-treatment, Treatment, and Post-treatment.

The Familiarization and Pre-treatment Trials: The hot-plate was activated and once it reached a temperature of $43.0^{\circ} \mathrm{C}$, subjects were instructed to immediately place their left forearm firmly on the hot-plate. Subjects were instructed to say "pain" when they first felt pain (threshold) and to say "stop" when the pain became too uncomfortable to continue (tolerance). Threshold and tolerance were recorded at the precise temperatures to which the subjects reported "pain" and "stop". To prevent injury, if the subjects did not say "stop" before the hotplate's temperature reached $48.0^{\circ} \mathrm{C}$, they were told to remove their arm from the heat. In addition, immediately following the removal of their arm, subjects were instructed to rate the intensity and unpleasantness of the pain at the instant that they reported "stop".

Following pain measurement, pre-treatment tactile sensitivity was measured. A graded series of calibrated nylon monofilaments (von Frey fibers) were applied to the area between the thumb and index finger on the dorsal side of the right hand (the hand not exposed to the heat). Tactile thresholds, defined as the minimal force required for the subject to detect a fibre on three consecutive trials, were estimated using a standard staircase method.

The Treatment Trial: Subjects in the Palatable, Neutral, and Unpalatable groups then consumed a chocolate chip cookie, a rice cake, or a black olive, respectively. Subjects in the Nothing (control) group did not consume anything. Instead, they were instructed to sit and read a selected passage from a psychology textbook for 5 min., the same amount of time allotted for the other groups to consume their food.

The Post-Treatment Trial: Immediately following treatment, subjects' pain and tactile sensitivity were assessed again by exposing them to the contact heat, followed by the monofilaments. After the experimental phase was complete, subjects in the treatment groups were asked to rate the palatability of the treatment food that they had consumed using a 10-point VAS (endpoints labelled "Strongly Like" and "Strongly Dislike"). This rating was obtained after the pain assessment rather than immediately after ingestion (i.e., before the pain assessment) in attempts to prevent Ss from guessing the experiment's hypothesis. Finally, all subjects completed a personal questionnaire intended to provide information about each subject's recent experience with factors known to modulate pain responsivity (e.g., phase of menstrual cycle, smoking, use of oral contraceptives) [26] [29]. All sessions were conducted between 1400 and $1700 \mathrm{~h}$ and lasted between 35 and 45 minutes.

\section{Results \& Discussion}

\subsection{Baseline Comparisons}

\subsubsection{Pain Measures}

To determine whether there were any relationships between the latency pain measures (threshold and tolerance), or between the VAS pain measures (intensity and unpleasantness), Pearson product-moment correlations were conducted across all participants. As expected, VAS ratings of pain intensity and unpleasantness were highly correlated at pre-treatment $(\underline{r}=0.40, \underline{p}<0.01)$ as were latency measures of pain threshold and tolerance $(\underline{r}=$ $0.75, \mathrm{p}<0.001)$.

\subsubsection{Pre-Treatment Group Differences}

One-way ANOVAs revealed no significant differences among the four groups for pre-treatment pain thresholds, pain tolerances, VAS ratings of intensity and unpleasantness, or tactile thresholds (all ps $>0.05$ ).

\subsection{Treatment Effects on Pain and Tactile Sensitivity}

One-way analyses of covariance (ANCOVAs) comparing the groups at post-treatment (with pre-treatment trial scores serving as the covariate) were performed on each of the four pain measures and on tactile thresholds. The analyses revealed that the groups differed on measures of pain tolerance $[\underline{F}(3,35)=4.71, \underline{p}=0.007]$ and unpleasantness $[\underline{F}(3,35)=2.98, \underline{p}=0.044]$. Post-hoc comparisons showed that the main effect on tolerance was 
accounted for by a difference between the Palatable sweet group and the Unpalatable group (Newman-Keuls, $\underline{\mathrm{p}}$ $<0.05)$. Figure 1 shows that pain tolerance was significantly greater for the Palatable group than the Unpalatable group. This figure also demonstrated that there were no significant differences in pain tolerance among the other groups. Surprisingly, for the unpleasantness ratings, both the Palatable and Neutral groups showed significantly higher ratings than the nothing group [Newman-Keuls, $\mathrm{p}<0.05$ ]. No significant group differences were found for the other 2 pain measures (both $\mathrm{ps}>0.05$ ) nor among tactile thresholds $[\mathrm{F}(3,35)=0.59, \mathrm{p}=0.623]$. Finally, omega-squared analyses revealed that the effect size for the significant results was 0.15 for unpleasantness, and 0.28 for tolerance, values which both indicate a "strong" experimental effect [30].

These results lend some support for the existence of SIA in human adult females. Relative to consuming an unpalatable food, consuming a palatable sweet food produced increased pain tolerance. Although the pain tolerance of the palatable and unpalatable groups did not differ significantly from the comparison groups (neutral and nothing), the respective rank order of the four pain tolerance scores was as consistent with the prediction that palatability is a critical factor (i.e., highest ranking for the Palatable group, lowest for the Unpalatable group, with both the Neutral and Nothing groups falling in between). Collectively, these findings confirm the hypothesis that the palatability of the ingested food is important for modifying pain perception. Further support for the role of palatability is provided by several other findings. First, we found significant positive correlations between palatability ratings and the changes from pre- to post-treatment in both pain tolerance $(\underline{r}=0.47, \underline{p}<0.01)$ and pain threshold $(\underline{\mathrm{r}}=0.36, \mathrm{p}<0.05)$. Second, as we reported previously [6], treatment groups differed on post-treatment unpleasantness ratings. Relative to the Nothing group, the sweet Palatable group and the Neutral group showed increased unpleasantness ratings following treatment.

\subsection{Secondary Analyses: Non-Treatment Effects}

\subsubsection{Food Palatability VAS Ratings}

To confirm that subjects were pre-assigned to the correct treatment groups (i.e., to ensure that those in the Palatable Group, considered the chocolate chip cookies palatable), following ingestion, subjects were asked to rate the food, using a 10-point VAS (endpoints labelled "Strongly Like" and "Strongly Dislike"). Following consumption, the mean VAS palatability ratings for each of the treatment foods were 8.70 (S.E.M. $=0.36$, range $=7$ - 10) for the cookies, 5.80 (S.E.M. $=1.80$, range $=2$ - 8) for the rice cakes, and 1.10 (S.E.M. $=0.10$, range $=1$ 2) for the black olives. A one-way ANOVA performed on the palatability ratings showed a significant Group effect $[\underline{F}(2,27)=97.83, \underline{p}<0.0001]$, with each treatment group differing from all other groups (Newman-Keuls. $\mathrm{p}<0.05)$. It is important also to note that the mean ratings of the different ingesta were virtually unchanged from those obtained at the outset of the experiment.

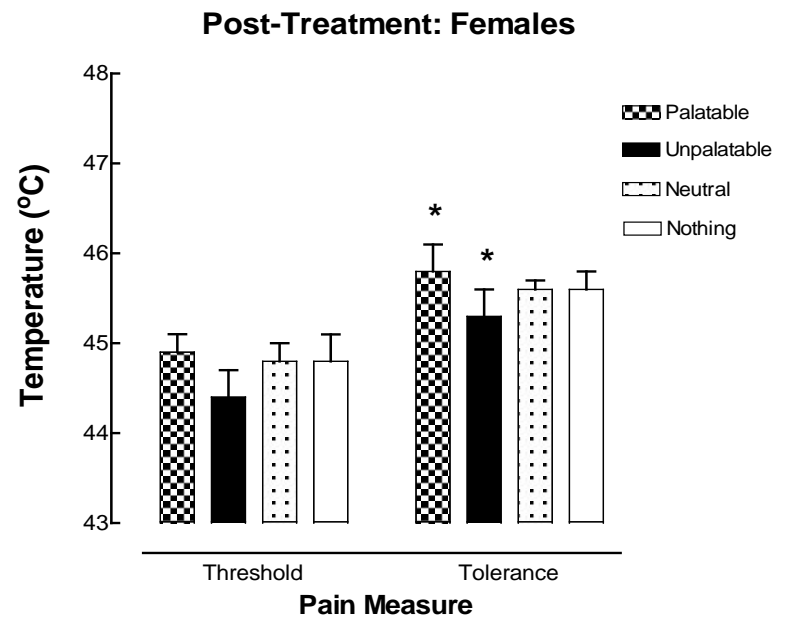

Figure 1. Experiment 1 post-treatment means (and standard error) for pain threshold and tolerance among females $(n=40)$ in each treatment group. The asterisk $\left(^{*}\right)$ denotes that the mean tolerance of the Palatable group differed significantly from the Unpalatable group $(\mathrm{p}<0.05)$. 


\subsubsection{Subject Variables}

To determine whether there were any relationships between either of the four pain measures and any of the recorded subject variables (e.g., age, stage of menstrual cycle, body weight, use of alcohol, cigarettes, illicit drugs, birth control pills and other medications, amount of sexual activity, exercise, sleep, and food/sweet consumption), multiple Pearson product-moment correlations were conducted. There were no significant correlations between the pain measures and any of the subject variables (all ps $>0.05$ ), suggesting that any pre-existing subject conditions had little, if any, effect upon the results.

\section{EXPERIMENT 2}

Experiment 1 showed that the contact-heat method is a suitable method for assessing SIA in adults, at least in women. Therefore, in an attempt to replicate and extend these findings, the present experiment again used the contact-heat method to assess the effects of sweet intake and palatability on analgesia, but this time with a greater number of subjects that included both males and females. Moreover, to examine further the role of palatability, and to maximize the possibility that subjects were experiencing the hedonic value of the palatable food, participants were allowed to consume as much of the experimental food as they desired.

\section{Material and Methods}

\subsection{Subjects}

Eighty (40 female and 40 male) right-handed, non-smoking, pain-free Caucasian university students served as subjects $(\mathrm{M}$ age $=21.9$ years old; range $=18-43$ years old $)$. An additional 2 subjects were tested but not included in the final sample; 1 female because it was later discovered that she did not meet the experiment's criteria (i.e., she was not appropriately food-deprived), and 1 male because he kept his forearm on the hot-plate at pre-treatment for the maximum temperature of $48.0^{\circ} \mathrm{C}$ (i.e., he showed maximum tolerance).

\subsection{Procedure}

This study used the same procedure as that of Experiment 1 with the following three exceptions. First, both males and females were tested. Second, before receiving a treatment food, the groups were matched according to their pre-treatment pain tolerance score. This was achieved by calculating for pre-treatment tolerance, a running group mean, including the subject currently being tested. Tolerance was chosen as the matching variable because it was the most sensitive measure both in Exp.1 as well as in previous reports [6] [7]. Third, rather than presenting the experimental subjects with only one serving of the treatment food, the subjects were offered either four chocolate-chip cookies, four rice cakes halves, or four black olives and were instructed to eat at least one serving and as much of the food as they wanted. The amount consumed by each subject was recorded.

\section{Results and Discussion}

\subsection{Baseline Comparisons}

\subsubsection{Pain Measures}

As in Expt. 1, intensity and unpleasantness measures were highly correlated at pre-treatment $(\underline{r}=0.67, \underline{p}<0.001$ as were measures of threshold and tolerance $(\underline{r}=0.67, \underline{p}<0.001)$.

\subsubsection{Treatment Group Differences}

As also reported in the previous experiment, one-way ANOVAs revealed no significant differences among the four groups at pre-treatment for pain threshold, pain tolerance, or intensity and unpleasantness VAS ratings (all ps $>0.05)$.

\subsubsection{Gender Differences}

Consistent with previous findings [6] [31] [32], one-way ANOVAs revealed significant gender differences for pre-treatment pain tolerance $[\underline{\mathrm{F}}(1,78)=12.07, \underline{\mathrm{p}}=0.0008]$, intensity $[\underline{\mathrm{F}}(1,78)=4.48, \underline{\mathrm{p}}=0.0375]$, and unpleasantness $[\underline{F}(1,78)=3.94, \underline{p}=0.0490]$. Interestingly, compared to men, women endured less heat (a differences of 0.7 deg. C), but they rated their pain as less intense and less unpleasant (a difference of $13 \%$ and $16 \%$, respec- 
tively). This may be explained by the fact that women left their arms on the hot-plate for shorter durations (tolerance) than did the men, and as a result, they may have perceived the pain as less intense and less unpleasant than men. Men and women did not differ significantly on either pain thresholds or on tactile thresholds (ANOVAs, both ps $>0.05$ ).

\subsection{Treatment Effects on Pain and Tactile Sensitivity}

For both men and women, one-way ANCOVAs comparing the Palatable, Unpalatable, Neutral and Nothing groups at post-treatment were performed on each of the 4 pain measures and on tactile thresholds. Women showed group differences on measures of pain threshold $[\underline{F}(3,34)=4.36, \underline{p}=0.0106]$ and pain tolerance $[\underline{F}(3,35)$ $=5.20, \mathrm{p}=0.0045]$. Specifically, post-hoc comparisons showed that the main effect on tolerance was accounted for by women in the Palatable group showing increased pain tolerance compared to women in all other groups (Newman-Keuls, $\mathrm{p}<0.05)$. As in Expt. 1, omega squared values were high $(0.23,0.32)$ again indicating strong experimental group effects [30]. Figure 2 shows that there were no differences in pain tolerance among the other groups.

For pain threshold however, the main effect was accounted for by a different pattern of results. Figure 2 shows that the post-treatment mean threshold of the Unpalatable group was significantly lower than all other groups (Newman-Keuls, $\mathrm{p}<0.05$ ), but did not differ from that of the Neutral group. The effect size was also found to be very strong (omega squared $=0.43$ ). No significant group differences were found for pain intensity or unpleasantness ratings (both ps $>0.05$ ) nor again among tactile measures $[F(3,75)=1.91, p>0.05]$. For men, there were no significant group differences for pain threshold, pain tolerance (see Figure 3), VAS ratings, or tactile thresholds (all ps $>0.05$ ).

As in our previous study with pressure algometry [6], the results indicate that the ingestion of a palatable sweet food produces analgesic effects in adult women, but probably not in adult men. Relative to women who consumed either unpalatable food, neutral food, or nothing, those who consumed palatable sweet food displayed increased pain tolerance. As in Experiment1 of the present study and in previous experiments [6], the posttreatment mean tolerance scores of the Palatable and Unpalatable groups differed the most (see Figure 2). Moreover, the post-treatment mean threshold of the Unpalatable group was lower than that of the Palatable and Nothing groups suggesting that the ingestion of an unpalatable food produces an effect on pain responsivity dif-

\section{Post-Treatment: Females}

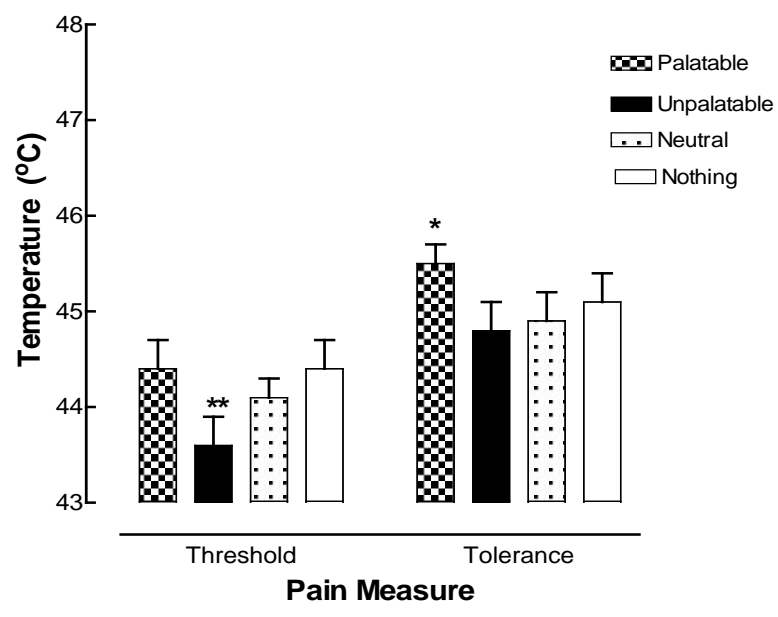

Figure 2. Experiment 2 post-treatment means (and standard error) for pain threshold and tolerance among females $(n=40)$ in each treatment group. The asterisk $(*)$ denotes that the mean tolerance of the Palatable group differed significantly from all other treatment groups (all $\mathrm{p}<0.05$ ). The double asterisk (**) denotes that the mean threshold of the Unpalatable group differed significantly from all other treatment groups (all $\mathrm{p}<0.05)$. 
Post-Treatment: Males

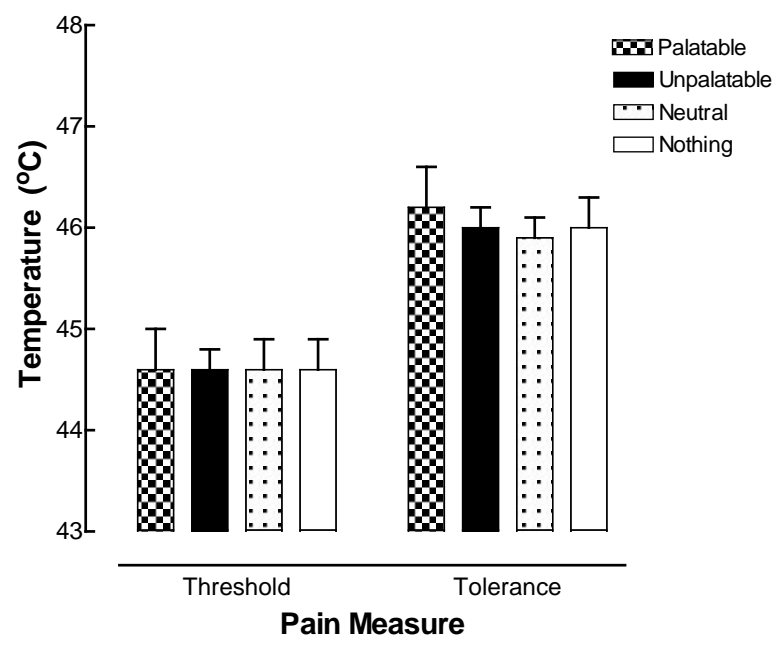

Figure 3. Experiment 2 post-treatment means (and standard error) for pain threshold and tolerance among males $(n=40)$ in each treatment group. There were no significant differences between any of the treatment groups (all $\mathrm{p}>0.05$ ).

ferent from that of palatable foods. Possible explanations for the relative hypoanalgesic effect of unpalatable foods are offered in section 6.3 of the General Discussion.

Collectively, these results implicate the importance of palatability in the analgesic effect of sweet ingesta. Moreover, the consistent finding that women's (but not men's) food palatability ratings are positively correlated with changes (from pre- to post-treatment) in pain tolerance $(\underline{r}=0.41, \underline{p}<0.01)$ and in pain threshold $(\underline{r}=0.50$, $\mathrm{p}<0.005$ ), also indicates that food palatability plays a critical role in the production of analgesia.

\subsection{Secondary Analyses: Non-Treatment Effects}

\subsubsection{Food Palatability VAS Ratings}

As in Expt. 1, to confirm that subjects were pre-assigned to the correct treatment groups, following ingestion, subjects were asked to rate the palatability of the food. The mean VAS palatability ratings for each of the treatment foods were 8.70 (S.E.M. $=0.40$, range $=6$ - 10) for the palatable cookies, 5.30 (S.E.M. $=0.5$, range $=3-8$ ) for the neutral rice cakes, and 1.40 (S.E.M. $=0.20$, range $=1-4$ ) for the unpalatable black olives. A two-way ANOVA [4 (Treatment Group) $\times 2$ (Gender)] performed on the palatability ratings showed a significant group effect $[\underline{\mathrm{F}}(2,54)=168.94, \underline{\mathrm{p}}<0.0001]$, with each treatment group differing from all other groups (Newman-Keuls. $\mathrm{p}<0.05$ ). Males and females did not differ on overall food palatability ratings $[\mathrm{F}(1,54)=0.51, \mathrm{p}=0.48]$, nor on individual food ratings [all ps $>0.05$ ]. Moreover, the women's palatability ratings did not differ between Expts. 1 and $2(\mathrm{p}<0.05)$, thus showing that eating "as much as they wanted", did not affect palatability ratings.

\subsubsection{Subject Variables}

Again, to determine whether there were any relationships between either of the four pain measures and any of the recorded subject variables (e.g., age, stage of menstrual cycle, body weight, use of alcohol, cigarettes, illicit drugs, birth control pills and other medications, amount of sexual activity, exercise, sleep, and food/sweet consumption), multiple Pearson product-moment correlations were conducted. There were no significant correlations between the pain measures and any of the subject variables (all ps $>0.05$ ), suggesting that any pre-existing subject conditions had little, if any, effect upon the results.

\section{General Discussion}

The present results provide two new significant findings. First, compared to previous pain induction methods such as cold pressor and pressure algometry, contact heat appears to be a superior method for assessing SIA in 
human adults. Contact heat data also shares the advantage of being more comparable to those obtained from previous well-controlled SIA studies with animals [9]-[11] [20]. Second, although present under some conditions, human SIA does not seem to persist robustly into adulthood, and/or it changes qualitatively from that observed in infancy. With development, the intensity of the analgesic effect appears to weaken substantially, it may be limited to females, and it appears to depend critically on food palatability rather than on sweet taste alone.

\subsection{Methodological and Measurement Issues}

The present study was the first to demonstrate that contact heat is a promising method for assessing the presence of SIA in human adults. In two separate experiments, women who consumed a highly rated sweet food (chocolate chip cookies) kept their forearm on a hot-plate longer than women who consumed either non-sweet foods (Expt $1 \& 2$ ) or nothing (Expt 2 only). Our results also suggest that contact heat may be a better method of experimental pain induction than those used previously (pressure algometry or CPT) as it does not appear to produce the ceiling effects that can mask the analgesic outcome of sweet ingestion. In the present study, fewer than $2 \%$ of the participants kept their arm on the hot-plate as it reached the maximum temperature, compared to 33\% of those who left a finger in the pressure algometer at maximum pressure [6], and 30\% to $35 \%$ who reached the maximum tolerance for the CPT [7] [23]. Secondly, contact heat pain is not cyclical in nature, but rather increases steadily over time. In contrast, cold pressor pain increases during the first minute, then slowly subsides, but increases again at various intervals later on [32] [33]. This periodicity compromises the interpretation of cold pain measures, especially for measurements beyond 60 seconds. Moreover, with the CPT, numbness quite frequently replaces the sensation of pain [34]. Thirdly, contact heat may be a superior method because both SIA and heat pain are believed to involve the endogenous opioid system. In rats, SIA to heat pain has been shown to be reversible by opioid antagonists [8] [9] [11]. In contrast, cold water immersion likely activates a non-opioid hormonal system [35] [36], and is therefore perhaps not as sensitive to sweets' analgesic effects. Low severity pain, such as that produced by heat (and perhaps pressure), may be more suitable for testing SIA than the high severity, cyclical, cold pressor pain. Therefore, contact heat not only reduces the potential for ceiling effects, but it also appears to activate opioid pathways, thus maximizing the likelihood of observing human SIA.

Concerning specific measures of pain, our studies of ingestion analgesia [present study; 6,7] consistently demonstrate that pain tolerance is the only index that reliably demonstrates the analgesic effect of sweet ingesta. Whether evaluating pain responsivity to contact heat, pressure, or cold water, threshold measures have shown inconsistent patterns, and intensity and unpleasantness VAS ratings have shown very mixed results (either no change or an increase in pain ratings-effects opposite to those expected for analgesia). It is possible that VAS ratings of unpleasantness and intensity are more suitable for assessing pain in clinical settings rather than for evaluations of experimental pain. Alternatively, VAS ratings may be preferable for evaluating tonic, rather than phasic, pain [6] [34] [37], or perhaps for low vs. high intensity pain [38]. Moreover, recent studies even question the validity of conventional VAS ratings for making comparisons across groups [39]. Note also that in all of our studies, the administration of pain and/or the ingestion of sweets have not produced any measurable changes in tactile thresholds, confirming our hypothesis that ingestion modulates pain pathways exclusively, rather than having a more general effect on somatosensory pathways. Therefore, in future experimental studies of ingestion analgesia, it may be unnecessary to record either tactile thresholds or VAS ratings of pain intensity and unpleasantness.

\subsection{SIA in Human Adults: A Modest Effect That Diminishes across Age and Gender}

The present finding of a modest effect of SIA in human adults is consistent with suggestions that SIA may disappear, or at least diminish, in adulthood [8] [23] [24], and/or it presents itself only under specific conditions. Our results also suggest that for adults, palatability rather than sweet taste, becomes the key determinant of SIA. We will expand upon these ideas in the sections below, beginning first with the developmental issue.

Anseloni and colleagues [8] found that intraoral sucrose produced analgesia to both mechanical and thermal stimuli in rat pups, but this effect declined after post-natal Day 10, and was absent after Day 17. Based on this work, they argued that SIA is limited to the pre-weaning period and then disappears when rats obtain the locomotor capacity to escape noxious stimuli. This developmental cessation or curtailment of SIA is also supported by data from human adults [23] [24] [34], including some of our own [6] [7]. However, numerous other studies 
of adult rats [10] [11] [40]-[42] have investigated the effects of chronic sucrose ingestion on analgesia and found that sweet-induced analgesia does persist with maturity. For example, sucrose produced analgesia in adult rats who consumed sucrose for 14 days, but not in rats who consumed sucrose for only 1 day [11]. Perhaps chronic sucrose ingestion is much more comparable to human adult sweet experiences than is the single intraoral sucrose infusion given to naïve rat pups or infants. It is possible that with age, human's experience with sweet ingestion (and pain) alters the inputs to the taste-analgesia circuitry. More specifically, the taste-induced analgesia system may be influenced increasingly by higher-order CNS processes [43] [44] and, as we argue below, may become influenced critically by food palatability.

The diminishing or the altering of human SIA with age appears to have a different course of development for males compared to females; specifically, it may disappear altogether in males. These developmental gender differences in pain sensitivity are likely due to both biological and psychosocial differences between males and females [31] [45] [46]. First, perhaps females, but not males, show SIA because of neuroanatomical differences in the pain, analgesia, and/or gustatory systems [47] [48]. For example, because of higher basal blood pressure levels which increase the levels of EOPs [48], men may show less sensitivity to pain and thus, a reduced potential for SIA. Alternatively, there may be gender differences in taste hedonics and its activation of reward and analgesia systems, or in the interpretation of pain and taste stimuli. For example, studies indicate that compared to females, males are less sensitive to sweet tastes [49], they have a reduced preference for sweets [50]-[52], and they have fewer sweet-sensitive cells in brainstem areas important in taste preference [53]. Therefore, sweets may taste less palatable to men, thus reducing their analgesic potential. Moreover, it is possible that there is some evolutionary adaptation for women, but not men, to retain SIA from infancy, perhaps due to the anticipated effects of the recurring and exclusively female "pain events", such as menstrual pain, pregnancy and childbirth [54].

The finding that SIA exists in adult women, but not men, does however conflict with those of other researchers [23] [24]. For example, Kakeda and Ishikawa [23] reported that after ingestion of a 24\% sweet solution, CPT pain thresholds increased in adult males, but not in females. Pepino \& Mennella [24] examined SIA in both 5 to 10 yr-old children and their mothers' responses to cold pressor pain and found that sucrose increased pain threshold and tolerance of children, but not those of women. These different findings may be attributed to a number of varying experimental parameters among the studies, namely method of pain induction, subject populations, and treatment (food palatability), but perhaps most importantly, by interpretation of the results. Considering first the methodological issues, we have argued above that the CPT used in most previous studies is not a suitable method for assessing SIA because of its potential for ceiling effects, its cyclical nature, and its' likely activation of non-opioid, rather than opioid, pathways. Additionally, the studies selected participants from different samples, namely Japanese students [23] vs. African-American mothers [24] vs. Caucasian students [6] [7]. Racial differences in both pain sensitivity [55]-[58] and taste preferences [59] have been well-established and might account for the differences among studies. Furthermore, the type of sweet ingesta chosen to evaluate SIA has differed across studies. Previous research has used pure sucrose solutions, which humans rarely consume, whereas our laboratory used more commonly ingested, realistic, and perhaps, more palatable foods (either soft drinks or chocolate chip cookies). If our hypothesis is correct that it is the palatability of the ingesta, rather than sweet content, that determines SIA, then this may explain the difference between genders, as several studies indicate that that highly concentrated sucrose solutions are preferred by men [60], but not by women [24] [34] [59] [61].

Finally, perhaps the most salient reason for the discrepant findings may be due to the interpretation of the results. Based on changes in pain threshold, but not pain tolerance, Kakeda and Ishikawa [23] reported that a rapid-onset, short-lasting SIA occurred in adult men. However, previous work with rats has shown that the analgesia produced by ingesting sweets is opioid mediated [9], and therefore, thought to affect the affectiveemotional component of pain (as measured by pain tolerance) as opposed to the sensory-discriminative component (as measured by threshold). It is likely that an analgesic effect shown only at threshold may not be indicative of true SIA. Ingestion analgesia occurs in 2 phases: an initial, short-lasting, "salience" phase (sometimes referred to as an orotactile or "distraction" effect) followed by a later, longer lasting "sweet/hedonic" phase (sometimes referred to an orogustatory effect) [12] [62]. Therefore, the highly concentrated sucrose solution [5] [23] used in previous work may have acted as a distractor, much like the brief effect produced by other "salient" ingesta, such as quinine or corn oil [12] [22] [62]. This transient, immediate analgesia may thus not be the typical opioid-mediated SIA like that evaluated here in the present work, but rather a nonopioid-mediated "distractor- 
induced analgesia”.

\subsection{Palatability}

The present study purposively manipulated the palatability of the ingesta in order to determine if palatability is a critical factor in the occurrence of analgesia, or whether the effect is due to sweetness alone, as the terminology "sweet-induced analgesia" has traditionally implied. Results from the present contact heat study, as well as those from our pressure algometry studies [6], suggest that palatability may be the key determinant in the occurrence of any ingestion-based analgesia, at least for female adults. More specifically, the present study reported that women who consumed a food that they rated as palatable (chocolate chip cookies), showed increased pain tolerance compared to those consuming either nothing (Expt. 2) or foods rated as neutral (Expt. 2) or unpalatable (Expts. 1 \& 2). Moreover, in both of the experiments reported here, the greatest differences in pain measures between groups were found between those consuming palatable vs. unpalatable foods. These group differences along with the positive correlations between palatability ratings and the changes in both pain threshold and tolerance following treatment, suggest that the analgesic effects of a sweet food may depend on its palatability. If this palatability argument is correct, this may explain why previous studies using sucrose solutions could not consistently demonstrate SIA in either adult women [23] [24] or men [7], as adults do not generally drink such highly-concentrated pure sucrose solutions.

This palatability explanation is supported by studies with rats and children. First, in a series of very well controlled experiments with rats, Foo and Mason [21] [63] demonstrated the importance of hedonic value in increasing rats' paw-lift latencies on a hot-plate. They found that water, sucrose, saccharin, and chocolate chips all produced analgesia, but when the hedonic value of these ingesta was reversed through conditioned taste aversion, the ingestion analgesia was attenuated. Similarly, they found that non-hedonic ingesta (quinine and $\mathrm{NaCl}$ ) did not induce analgesia. However, if the hedonic value of the quinine and $\mathrm{NaCl}$ was changed from "bad" to "good" by depriving rats of those ingesta, an increase of rats' paw-lift latencies was observed. They concluded that in order for analgesia to occur, food has to be both palatable and hedonically positive. These behavioral findings are supported by physiological changes in rat brain and plasma. For example, Yamamoto, Sako and Maeda [3] found that the release of beta-endorphin in CSF and plasma is positively correlated with the palatability of taste stimuli. Secondly, the importance of palatability has been demonstrated in both human infants and older children. Pepino and Mennella [24] reported that 5 - 10 yr-old children (though not adults) who preferred higher concentrations of sucrose showed greater analgesia to pain than children who preferred lower sucrose concentrations, again suggesting that sucrose's efficacy may be related to its hedonic value. With newborn infants, Blass and Smith [17] compared the effects of four different sugars (sucrose, fructose, glucose and lactose) and water on crying and found that the most preferred sugars (sucrose and fructose) [64] produced the greatest calming effects. Similarly, compared to water, both sucrose and aspartame, but not polycose (a non-sweet carbohydrate), reduced crying in newborn infants [12]. The authors attributed this effect to the sweetness or positive hedonic value of the sweet ingesta.

Collectively, these findings demonstrate that palatability may be a critical factor in producing ingestion analgesia. Upon closer inspection of the present results, it appears that the type of ingesta may play a modulatory, rather than strictly analgesic role, in pain perception. The present study reported that the largest and most consistent difference in tolerance occurred between the Palatable and Unpalatable groups. Moreover, the pain threshold of the Unpalatable group was lower than that of the Palatable, Neutral and Nothing groups. It is an interesting possibility that unpalatable foods may decrease opioid activity whereas palatable foods increase opioid activity. Alternatively, unpalatable foods may indirectly affect the opioid system by modulating the activity of another neurotransmitter or neuromodulator involved in both food ingestion and pain modulation, such as the neuropeptide, cholecystokinin (CCK). CCK has been shown to attenuate both eating and morphine analgesia [65] [66]. These effects are thought to be mediated, at least in part, by CCK's influence on opioid systems that potentiate eating [67]. Therefore, in the present study, the consumption of unpalatable foods may have released CCK into the CNS where it then interacted with opioid mechanisms, blocking opioid action and thus producing a transient hypoanalgesia.

\section{Conclusion}

In summary, the present results confirm the importance of palatability in producing what has been referred to as 
"sweet-induced analgesia". Moreover, the present findings are the first to demonstrate that contact heat is a suitable, if not superior, method for the assessment of ingestion analgesia in human adults, at least among females. Women who ingest palatable food endure contact heat pain for longer durations than women who consume nothing, or foods rated as unpalatable or neutral. Therefore, it appears that ingestion analgesia, or perhaps more accurately, palatability-induced analgesia (PIA), does persist beyond infancy. However, the phenomenon may not be as pronounced in adulthood, as it appears to reduce in intensity, is observable only in women, and is highly dependent upon food palatability. This relative suppression may be explained by a decrease in the hedonic value of food, perhaps due to increased variety and experience, or by developmental changes in our pain and analgesia systems (e.g., either chronically elevated opioid levels and/or a down- regulation of opioid receptors, as a result of greater experience with pain and sweets). It may be that PIA (especially in men) serves little biological advantage for human adults compared to infants in whom PIA may serve as mediator of important behaviours such as mother-infant attachment, appropriate food selection, and/or energy conservation.

\section{Acknowledgements}

This research was supported by Natural Sciences and Engineering Research Council of Canada (NSERC) operating grant (A1221) to M. Holder, and by fellowships from NSERC and the Women's Association of Memorial University to M. Mercer.

\section{References}

[1] Dum, J., Gramsch, C. and Herz, A. (1983) Activation of Hypothalamic Beta-Endorphin Pools by Reward Induced by Highly Palatable Food. Pharmacology Biochemistry and Behavior, 18, 443-447. http://dx.doi.org/10.1016/0091-3057(83)90467-7

[2] Melchior, J.C., Rigaud, D., Colas-Linhart, N., Petiet, A., Girard, A. and Apfelbaum, M. (1991) Immunoreactive BetaEndorphin Increases after an Aspartame Chocolate Drink in Healthy Human Subjects. Physiology \& Behavior, 50, 941-944. http://dx.doi.org/10.1016/0031-9384(91)90418-n

[3] Yamamoto, T., Sako, N. and Maeda, S. (2000) Effects of Taste Stimulation on Beta-Endorphin Levels in Rat Cerebrospinal Fluid and Plasma. Physiology \& Behavior, 69, 345-350. http://dx.doi.org/10.1016/S0031-9384(99)00252-8

[4] Yamamoto, T. (2003) Brain Mechanisms of Sweetness and Palatability of Sugars. Nutrition Reviews, 61, S5-S9. http://dx.doi.org/10.1301/nr.2003.may.S5-S9

[5] Kakeda, T. (2010) Potential of Sucrose-Induced Analgesia to Relieve Pain in Male Adults: A Preliminary Study. Japan Journal of Nursing Science, 7, 169-173. http://dx.doi.org/10.1111/j.1742-7924.2010.00150.x

[6] Mercer, M.E. and Holder, M.D. (1997) Antinociceptive Effects of Palatable Sweet Ingesta on Human Responsivity to Pressure Pain. Physiology \& Behavior, 61, 311-318. http://dx.doi.org/10.1016/s0031-9384(96)00400-3

[7] Mercer, M.E. and Holder, M.D. (2013) Assessing the Analgesic Effects of Sucrose to Cold Pressor Pain in Human Adults. Journal of Behavioral and Brain Science, 3, 7-12. http://dx.doi.org/10.4236/jbbs.2013.31002

[8] Anseloni, V.C., Weng, H.R., Terayama, R., Letizia, D., Davis, B.J., Ren, K., et al. (2002) Age-Dependency of Analgesia Elicited by Intraoral Sucrose in Acute and Persistent Pain Models. Pain, 97, 93-103. http://dx.doi.org/10.1016/S0304-3959(02)00010-6

[9] Blass, E.M., Fitzgerald, E. and Kehoe, P. (1987) Interactions between Sucrose, Pain and Isolation Distress. Pharmacology Biochemistry and Behavior, 26, 483-489. http://dx.doi.org/10.1016/0091-3057(87)90153-5

[10] Holder, M.D. (1988) Responsivity to Pain in Rats Changed by the Ingestion of Flavoured Water. Behavioral and Neural Biology, 49, 45-53. http://dx.doi.org/10.1016/S0163-1047(88)91207-1

[11] Segato, F.N., Castro-Souza, C., Segato, E.N., Morato, S. and Coimbra, N.C. (1997) Sucrose Ingestion Causes Opioid Analgesia. Brazilian Journal of Medical and Biological Research, 30, 981-984. http://dx.doi.org/10.1590/S0100-879X1997000800011

[12] Barr, R.G., Pantel, M.S., Young, S.N., Wright, J.H., Hendricks, L.A. and Gravel, R. (1999) The Response of Crying Newborns to Sucrose: Is It a "Sweetness” Effect? Physiology \& Behavior, 66, 409-417. http://dx.doi.org/10.1016/S0031-9384(98)00294-7

[13] Blass, E.M. and Hoffmeyer, L.B. (1991) Sucrose as an Analgesic for Newborn Infants. Pediatrics, 87, $215-218$.

[14] Smith, B.A., Fillion, T.J. and Blass, E.M. (1990) Orally-Mediated Sources of Calming in 1- to 3-Day-Old Human Infants. Developmental Psychology, 26, 731-737. http://dx.doi.org/10.1037/0012-1649.26.5.731

[15] Stevens, B., Yamada, J. and Ohlsson, A. (2010) Sucrose for Analgesia in Newborn Infants Undergoing Painful Proce- 
dures. Cochrane Database of Systematic Reviews, No. 1, Article ID: CD001069. http://dx.doi.org/10.1002/14651858.cd001069.pub3

[16] Ramenghi, L.A., Evans, D.J. and Leave, M.I. (1999) "Sucrose Analgesia”: Absorptive Mechanism or Taste Perception? Archives of Disease in Childhood —Fetal and Neonatal Edition, 80, F146-F147. http://dx.doi.org/10.1136/fn.80.2.F146

[17] Blass, E.M. and Smith, B.A. (1992) Differential Effects of Sucrose, Fructose, Glucose, and Lactose on Crying in 1- to 3-Day-Old Human Infants: Qualitative and Quantitative Considerations. Developmental Psychology, 28, 804-810. http://dx.doi.org/10.1037/0012-1649.28.5.804

[18] Harrison, D., Stevens, B., Bueno, M., Yamada, J., Adams-Webber, T., Beyene, J. and Ohlsson, A. (2010) Efficacy of Sweet Solutions for Analgesia in Infants between 1 and 12 Months of Age: A Systematic Review. Archives of Disease in Childhood, 95, 406-413. http://dx.doi.org/10.1136/adc.2009.174227

[19] Blass, E.M. and Fitzgerald, E. (1988) Milk-Induced Analgesia and Comforting in 10-Day-Old Rats: Opioid Mediation. Pharmacology Biochemistry and Behavior, 29, 9-13. http://dx.doi.org/10.1016/0091-3057(88)90266-3

[20] Blass, E.M. and Shide, D.J. (1994) Some Comparisons among the Calming and Pain-Relieving Effects of Sucrose, Glucose, Fructose and Lactose in Infant Rats. Chemical Senses, 19, 239-249. http://dx.doi.org/10.1093/chemse/19.3.239

[21] Foo, H. and Mason, P. (2009) Analgesia Accompanying Food Consumption Requires Ingestion of Hedonic Foods. Journal of Neuroscience, 29, 13053-13062. http://dx.doi.org/10.1523/JNEUROSCI.3514-09.2009

[22] Shide, D.J. and Blass, E.M. (1989) Opioidlike Effects of Intraoral Infusions of Corn Oil and Polycose on Stress Reactions in 10-Day-Old Rats. Behavioral Neuroscience, 103, 1168-1175. http://dx.doi.org/10.1037/0735-7044.103.6.1168

[23] Kakeda, T. and Ishikawa, T. (2011) Gender Differences in Pain Modulation by a Sweet Stimulus in Adults: A Randomized Study. Nursing \& Health Sciences, 13, 34-40. http://dx.doi.org/10.1111/j.1442-2018.2010.00573.x

[24] Pepino, M.Y. and Mennella, J.A. (2005) Sucrose-Induced Analgesia Is Related to Sweet Preferences in Children but Not Adults. Pain, 119, 210-218. http://dx.doi.org/10.1016/j.pain.2005.09.029

[25] Vaswani, K.K. and Tejwani, G.A. (1986) Food Deprivation-Induced Changes in the Level of Opioid Peptides in the Pituitary and Brain of Rat. Life Sciences, 38, 197-201. http://dx.doi.org/10.1016/0024-3205(86)90012-3

[26] Pomerlau, O.F., Turk, D.C. and Fertig, J.B. (1984) The Effects of Cigarette Smoking on Pain and Anxiety. Addictive Behaviors, 9, 265-271. http://dx.doi.org/10.1016/0306-4603(84)90018-2

[27] Johnson, S. (1974) The Evaluation of Pain in Man. Pain, 2, 1-3.

[28] Price, D.D., McGrath, P.A., Rafii, A. and Buckingham, B. (1983) The Validation of Visual Analogue Scales as Ratio Scale Measures for Chronic and Experimental Pain. Pain, 17, 45-56. http://dx.doi.org/10.1016/0304-3959(83)90126-4

[29] Goolkasian, P. (1980) Cyclic Changes in Pain Perception: An ROC Analysis. Perception \& Psychophysics, 27, 499504. http://dx.doi.org/10.3758/BF03198677

[30] Keppel, G. and Wilkins, T. (2007) Design and Analysis. 5th Edition, Prentice Hall, Upper Saddle River.

[31] Wise, E.A., Price, D.D., Myers, C.D., Heft, M.W. and Robinson, M.E. (2002) Gender Role Expectations of Pain: Relationship to Experimental Pain Perception. Pain, 96, 335-342. http://dx.doi.org/10.1016/S0304-3959(01)00473-0

[32] Feine, J.S., Bushnell, M.C., Miron, D. and Duncan, G.H. (1991) Sex Differences in the Perception of Noxious Heat Stimuli. Pain, 44, 255-262. http://dx.doi.org/10.1016/0304-3959(91)90094-E

[33] Wolf, S. and Hardy, J.D. (1941) Studies on Pain. Observations on Pain Due to Local Cooling and on Factors Involved in the “Cold Pressor” Effect. Journal of Clinical Investigation, 20, 521-533. http://dx.doi.org/10.1172/JCI101245

[34] Miller, A., Barr, R.G. and Young, S.N. (1994) The Cold Pressor Test in Children: Methodological Aspects and the Analgesic Effect of Intraoral Sucrose. Pain, 56, 175-183. http://dx.doi.org/10.1016/0304-3959(94)90092-2

[35] Mayer, D.J. and Watkins, L.R. (1984) Multiple Endogenous Opiate and Nonopiate Analgesia Systems. Advances in Pain Research and Therapy, 6, 253-276.

[36] Terman, G.W., Morgan, M.J. and Liebeskind, J.C. (1986) Opioid and Non-Opioid Stress Analgesia from Cold Water Swim: Importance of Stress Severity. Brain Research, 372, 167-171. http://dx.doi.org/10.1016/0006-8993(86)91472-1

[37] Houle, M., McGrath, P.A., Moran, G. and Garrett, O.J. (1988) The Efficacy of Hypnosis- and Relaxation-Induced Analgesia on Two Dimensions of Pain for Cold Pressor and Electrical Tooth Pulp Stimulation. Pain, 33, 241-251. http://dx.doi.org/10.1016/0304-3959(88)90096-6

[38] Feine, J.S., Bushnell, M.C. and Duncan, D.H. (1988) Within-Subject Measurements of Heat Pain: Comparison of Magnitude Matching and VAS. Canadian/American Pain Society Abstract, 1, SS-5c.

[39] Dionne, R.A., Bartoshuk, L., Mogil, J. and Witter, J. (2005) Individual Responder Analyses for Pain: Does One Pain 
Scale Fit All? Trends in Pharmacological Sciences, 26, 125-130. http://dx.doi.org/10.1016/j.tips.2005.01.009

[40] Bergmann, F., Lieblich, I., Cohen, E. and Ganchrow, J.R. (1985) Influence of Intake of Sweet Solutions on the Analgesic Effect of a Low Dose of Morphine in Randomly Bred Rats. Behavioral and Neural Biology, 44, 347-353. http://dx.doi.org/10.1016/S0163-1047(85)90616-8

[41] Lieblich, I., Cohen, E., Ganchrow, J.R., Blass, E.M. and Bergmann, F. (1983) Chronically Elevated Intake of Saccharin Solution Induces Morphine Tolerance in Genetically Selected Rats. Science, 221, 871-873. http://dx.doi.org/10.1126/science.6879185

[42] D’Anci, K.E., Kanarek, R.B. and Marks-Kaufman, R. (1997) Beyond Sweet Taste: Saccharin, Sucrose, and Polycose Differ in Their Effects upon Morphine-Induced Analgesia. Pharmacology Biochemistry and Behavior, 56, 341-345. http://dx.doi.org/10.1016/S0091-3057(96)00227-4

[43] Kelley, A.E., Baldo, B.A., Pratt, W.E. and Will, M.J. (2005) Corticostriatal-Hypothalamic Circuitry and Food Motivation: Integration of Energy, Action and Reward. Physiology \& Behavior, 86, 773-795. http://dx.doi.org/10.1016/j.physbeh.2005.08.066

[44] Leknes, S. and Tracey, I. (2008) A Common Neurobiology for Pain and Pleasure. Nature Reviews Neuroscience, 9, 314-320. http://dx.doi.org/10.1038/nrn2333

[45] Mogil, J.S. and Bailey, A.L. (2011) Sex and Gender Differences in Pain and Analgesia. Progress in Brain Research, 186, $141-157$.

[46] Wiesenfeld-Hallin, Z. (2005) Sex Differences in Pain Perception. Gender Medicine, 2, 137-145. http://dx.doi.org/10.1016/S1550-8579(05)80042-7

[47] Bartoshuk, L.M., Duffy, V.B. and Miller, I.J. (1994) PTC/PROP Tasting: Anatomy, Psychophysics, and Sex Effects. Physiology \& Behavior, 56, 1165-1171. http://dx.doi.org/10.1016/0031-9384(94)90361-1

[48] Lewkowski, M.D., Ditto, B., Roussos, M. and Young, S.N. (2003) Sweet Taste and Blood Pressure-Related Analgesia. Pain, 106, 181-186. http://dx.doi.org/10.1016/S0304-3959(03)00333-6

[49] Valenstein, E.S., Kakolewski, J.W. and Cox, V.C. (1967) Sex Differences in Taste Preference for Glucose and Saccharin Solutions. Science, 156, 942-943. http://dx.doi.org/10.1126/science.156.3777.942

[50] Drenowski, A., Krahn, D.D., Demitrack, M.A., Nairn, K. and Gosnell, B.A. (1992) Taste Responses and Preferences for Sweet High-Fat Foods: Evidence of Opioid Involvement. Physiology \& Behavior, 51, 371-379. http://dx.doi.org/10.1016/0031-9384(92)90155-U

[51] Grogan, S.C., Bell, R. and Conner, M. (1997) Eating Sweet Snacks: Gender Differences in Attitudes and Behavior. Appetite, 28, 19-31. http://dx.doi.org/10.1006/appe.1996.0067

[52] Velle, W. (1987) Sex Differences in Sensory Functions. Perspectives in Biology and Medicine, 30, 490-522. http://dx.doi.org/10.1353/pbm.1987.0015

[53] Di Lorenzo, P.M. and Monroe, S. (1989) Taste Responses in the Parabrachial Pons of Male, Female and Pregnant Rats. Brain Research Bulletin, 23, 219-227. http://dx.doi.org/10.1016/0361-9230(89)90151-2

[54] Unruh, A.M. (1996) Gender Variations in Clinical Pain Experience. Pain, 65, 123-167. http://dx.doi.org/10.1016/0304-3959(95)00214-6

[55] Edwards, C.L., Fillingim, R.B. and Keefe, F. (2001) Race, Ethnicity and Pain. Pain, 94, 133-137. http://dx.doi.org/10.1016/S0304-3959(01)00408-0

[56] Komiyama, O., Kawara, M. and De Laat, A. (2007) Ethnic Differences regarding Tactile and Pain Thresholds in the Trigeminal Region. The Journal of Pain, 8, 363-369. http://dx.doi.org/10.1016/j.jpain.2006.12.002

[57] Rahim-Williams, F.B., Riley III, J.L., Herrera, D., Campbell, C., Hastie, B.A. and Fillingim, R.B. (2007) Ethnic Identity Predicts Experimental Pain Sensitivity in African Americans and Hispanics. Pain, 129, 177-184. http://dx.doi.org/10.1016/j.pain.2006.12.016

[58] Woodrow, K.M., Friedman, G.D., Siegelaub, B. and Collen, M.F. (1972) Pain Tolerance: Differences According to Age, Sex and Race. Psychosomatic Medicine, 34, 548-556. http://dx.doi.org/10.1097/00006842-197211000-00007

[59] Pepino, M.Y. and Mennella, J.A. (2005) Factors Contributing to Individual Differences in Sucrose Preference. Chemical Senses, 30, i319-i320. http://dx.doi.org/10.1093/chemse/bjh243

[60] Enns, M.P., Van Itallie, T.B. and Grinker, J.A. (1979) Contributions of Age, Sex and Degree of Fatness on Preferences and Magnitude Estimations for Sucrose in Humans. Physiology \& Behavior, 22, 999-1003. http://dx.doi.org/10.1016/0031-9384(79)90346-9

[61] Desor, J.A. and Beauchamp, G.K. (1987) Longitudinal Changes in Sweet Preferences in Humans. Physiology \& Behavior, 39, 639-641. http://dx.doi.org/10.1016/0031-9384(87)90166-1

[62] Graillon, A., Barr, R.G., Young, S.N., Wright, J. and Hendricks, L.A. (1997) Differential Response to Intraoral Sucrose, 
Quinine and Corn Oil in Crying Human Newborns. Physiology \& Behavior, 62, 317-325. http://dx.doi.org/10.1016/S0031-9384(97)88987-1

[63] Foo, H. and Mason, P. (2011) Ingestion Analgesia Occurs When a Bad Taste Turns Good. Behavioral Neuroscience, 125, 956-961. http://dx.doi.org/10.1037/a0025542

[64] Desor, J.A., Maller, O. and Turner, R.E. (1973) Taste in Acceptance of Sugars by Human Infants. Journal of Comparative and Physiological Psychology, 84, 496-501. http://dx.doi.org/10.1037/h0034906

[65] Faris, P.L., Komisaruk, B., Watkins, L.R. and Mayer, D.J. (1983) Evidence for the Neuropeptide Cholecystokinin as an Antagonist of Opiate Analgesia. Science, 219, 310-312. http://dx.doi.org/10.1126/science.6294831

[66] Watkins, L.R., Kinscheck, I.B., Kaufman, E.F.S., Miller, J., Frenk, H. and Mayer, D.J. (1985) Cholecystokinin Antagonists Selectively Potentiate Analgesia Induced by Endogenous Opiates. Brain Research, 327, 181-190. http://dx.doi.org/10.1016/0006-8993(85)91512-4

[67] Leibowitz, S.F. and Stanley, B.G. (1986) Neurochemical Controls of Appetite. In: Ritter, R.C., Ritter, S., Barnes, C.D., Eds., Feeding Behavior Neural and Humoral Controls, Academic Press, New York, 191-234. http://dx.doi.org/10.1016/b978-0-12-589060-1.50012-2 